\title{
EFECTO DE LA COMPOSICIÓN DEL GAS DE REFINERÍA SOBRE LAS CARACTERÍSTICAS DEL PROCESO DE COMBUSTIÓN
}

\author{
O. M. Cala* \\ L. Meriño** \\ V. Kafarov ${ }^{* * *}$ \\ J. Saavedra***
}

Recibido: 20/05/2013

Aprobado: 25/10/2013

\section{RESUMEN}

En este artículo de investigación científica se analiza el efecto del cambio de la composición del gas combustible (Gas de Refinería (GR) por Gas Natural (GN)) sobre las características del proceso combustión en hornos de la industria de refinación del petróleo; se evaluó el poder calorífico, el índice de Wobbe (IW) y exceso de oxígeno, para mezclas combustibles de composición variable. Mediante simulación computacional del proceso de combustión se calculó la temperatura adiabática de llama, eficiencia y la composición de los productos de combustión. Se evaluaron mezclas de gases combustibles con poderes caloríficos entre $800-2500 \mathrm{Btu} / \mathrm{pie}^{3}$ y se compararon con la combustión de gas natural. Se registró variabilidad en la temperatura adiabática y la eficiencia en función de la composición del gas y el exceso de oxígeno, lo que genera inestabilidad en el horno y mayor impacto ambiental.

Palabras clave: gas de refinería, combustión, eficiencia energética, combustible.

Oscar Mauricio Cala. Ingeniero Químico, Centro de Investigación para el Desarrollo Sostenible en Industria y Energía (CIDES), Universidad Industrial de Santander. Cra 27 calle 9 ciudad universitaria, Bucaramanga, Colombia. Tel. 6344000 Ext: 2603, E-mail: oscar.cala@correo. uis.edu.co

** Lourdes Meriño Stand, MSc en Ingeniería Química, Candidata a doctor, Centro de Investigación para el Desarrollo Sostenible en Industria y Energía (CIDES), Universidad Industrial de Santander. Cra 27 calle 9 ciudad universitaria, Bucaramanga, Colombia. Tel. 6344000 Ext: 2603, E-mail: lourdes.merino@correo.uis.edu.co

*** Viatcheslav Kafarov, P.h.D. Docente de planta, Escuela de Ingeniería Química, Director del Centro de Investigación para el Desarrollo Sostenible en Industria y Energía (CIDES), Universidad Industrial de Santander. Cra 27 calle 9 ciudad universitaria, Bucaramanga, Colombia. Tel.6344000.email: kafarov@uis.edu.co

**** Jaqueline Saavedra Rueda, P.h. D. en Ingeniería Química, Líder de plantas piloto, Instituto Colombiano del Petróleo - ECOPETROL Km. 7 Vía a Piedecuesta, Colombia. Tel: +57 (7) 6847331. email: jaqueline.saavedra@ecopetrol.com.co 


\title{
EFFECT OF REFINERY GAS COMPOSITION ON CHARACTERISTICS OF COMBUSTION PROCESS
}

\begin{abstract}
This scientific research article is an analysis of the effect fuel gas composition change (from Refinery Gas to Natural Gas) has on the characteristics of combustion process in industrial oil refining furnaces; heat power, Wobbe Index, and oxygen excess were evaluated for combustible mixtures of variable composition. Through computational simulation of the combustion process, adiabatic temperature of the flame, efficiency, and composition of combustion products were calculated. Mixtures of combustible gases with heat powers between 800 and $2500 \mathrm{Btu} / \mathrm{cubic}$ feet were evaluated and compared to natural gas combustion. Variability of adiabatic temperature and efficiency in function of gas composition and oxygen excess occurred; and this resulted in furnace instability and higher environmental impact.
\end{abstract}

Key words: Refinery gas; combustion; energy efficiency; fuel. 


\section{INTRODUCCIÓN}

Actualmente el mundo se está enfrentado a grandes retos causados por el desarrollo económico global, que se reflejan en un incremento en la demanda de energía mundial y el aumento de las emisiones de dióxido de carbono [1]. En una planta química, tal como una refinería, la energía representa el $40 \%$ del costo total de operación, por lo cual la industria petroquímica ha venido implementando estrategias para aumentar la eficiencia energética, entre las que se destacan: el fortalecimiento de la gestión de la energía, el cambio de las fuentes de energía y la recuperación y posterior reutilización de residuos de energía [2]. Actualmente, los procesos petroquímicos que se desarrollan en una refinería utilizan como fuente principal de energía una mezcla de gas natural $(\mathrm{GN})$ y gas gastado de proceso, el cual es recuperado de los distintos procesos (Unidad de cracking catalítico, unidad de desulfurización y unidad de reformado catalítico) y reutilizado como sustituto del gas natural, y se le denomina gas de refinería (GR); dicho gas presenta una composición que variará ampliamente dependiendo del proceso del cual provenga, con altos contenidos de hidrógeno, etileno, propano y propileno.

Este tipo de sustitución en la fuente del combustible ha sido estudiada desde el siglo anterior y se ha encontrado una amplia diversidad de compuestos que pueden ser utilizados en procesos de combustión dependiendo de la fuente de origen y su disponibilidad; de esta manera surgió el término intercambiabilidad de gases el cual hace referencia a la posibilidad de remplazar un combustible por otro sin que se afecten las características de operación del aparato doméstico, comercial o industrial [3]. Wobbe [4] presentó "el Índice de Wobbe" (poder calorífico dividido entre la raíz cuadrada de la gravedad específica), el cual describe la habilidad de un gas para liberar calor al quemador como una función de su densidad. De igual forma surge una diversidad de índices para evaluar la intercambiabilidad: como los índices de American Gas Association [5], el método de Delbourg [6], y el
Factor de Weaver [7] entre los más importantes; sin embargo, en dichos estudios no se tiene en cuenta el gas de refinería.

Recientemente los investigadores se han centrado en el gas de refinería y han estudiado este cambio de combustible como una manera de reducir el costo de la energía Hsieh analizó la influencia de utilizar un gas de refinería rico en hidrógeno $(50-80 \%$ mol) y encontró una reducción en las emisiones de $\mathrm{CO}_{2}$ y $\mathrm{NO}_{x}$ del 16,4 y 8,2 $\%$ respectivamente [8]. Otros estudios analizan las emisiones de $\mathrm{NO}$ y $\mathrm{CO}_{2}$ utilizando diferente relación combustible/gas de refinería, rico en hidrógeno, en calderas de media y alta presión, cuyos resultados muestran que se puede ahorrar costos en el combustible y reducir las emisiones de gases de efecto invernadero utilizando el GR $[9,10]$, todas estas investigaciones se basan en el hecho de que el hidrógeno es catalogado como un combustible limpio por su poder calorífico sin la generación de contaminantes o residuos [11]. Otro parámetro a analizar con el GR es el exceso de aire, pues afecta tanto la eficiencia térmica como a los impactos ambientales generados en los hornos [12]. Cuando el exceso de oxígeno es elevado, se incrementa la concentración de $\mathrm{O}_{2}$ en el área de combustión que conduce a un aumento de la temperatura de llama, y por tanto, una caída de temperatura en el área de radiación (Zona de reacción) que disminuye la eficiencia del horno [13]. La alta temperatura de llama, junto con combustión turbulenta en el horno, causa la reacción del oxígeno con el nitrógeno, formando $\mathrm{NO}$ (óxido nítrico) y $\mathrm{NO}_{2}$ (Dióxido de nitrógeno) [14]. De igual forma, al emplear bajos excesos de aire se obtiene un incremento en la eficiencia térmica, pero se corre el riesgo de tener mezclas poco íntimas de aire y combustible que pueden ocasionar combustión incompleta [15], por lo cual es necesario determinar el valor óptimo de oxígeno a utilizar en todo proceso combustión.

Sin embargo, las investigaciones mencionadas anteriormente utilizan GR con alto contenido de 
hidrógeno (50-80\%), pero en los procesos petroquímicos dichos gases se producen en menor cantidad, por lo cual este estudio se enfoca en simular varias corrientes de GR con diferentes composiciones, las cuales son recuperadas y alimentadas a un horno de combustión, con el objetivo de evaluar la influencia de usar el GR sobre las propiedades fisicoquímicas de los productos de la combustión.

\section{MATERIALESY MÉTODOS}

Las reacciones de combustión, utilizando como combustible el gas de refinería, fueron simuladas mediante modelos químicos y termodinámicos usando el software comercial Aspen Hysys 2006.5. Para el cálculo de las propiedades fisicoquímicas de las corrientes de GN, GR y aire se seleccionó la ecuación de estado de Peng Robinson pues es el paquete de fluidos recomendado para aplicaciones en el gas, petróleo y petroquímica, ya que se garantiza la precisión de la misma para una gran variedad de sistemas en un amplio rango de condiciones con alto grado de eficiencia y confiabilidad. La metodología planteada para la simulación consta de las siguientes etapas:

\subsection{Revisión de antecedentes históricos y selección de componentes a evaluar}

El primer paso para realizar la simulación del proceso de combustión consistió en la selección de los componentes presentes en el GR; para ello se realizó la revisión de los datos históricos de aproximadamente 120 cromatografías pertenecientes a corrientes de GR provenientes de distintas unidades de una refinería. Posteriormente se procedió a seleccionar una mezcla representativa del GR, mediante un análisis estadístico realizado por el software Statgraphics, utilizando como criterio base el PCI.

\subsection{Evaluación de propiedades}

La composición del gas combustible genera impactos significativos sobre la operación y la vida útil de los equipos de combustión (hornos y calderas), pues puede variar de tal forma que modifica el poder calorífico de la mezcla de combustible presentándose un amplio rango que va desde $800-2500 \mathrm{Btu} /$ pie $^{3}$. Para medir el efecto de la composición sobre el proceso de combustión, se estudiaron algunas variables influyentes en el proceso, tales como el poder calorífico inferior, el índice de wobbe, la temperatura adiabática de llama, exceso de oxígeno, composición de los productos de combustión y la eficiencia del proceso. Todo esto como una herramienta en la toma de decisiones en cuanto al uso del gas de refinería.

\subsection{Poder calorífico inferior $(\mathrm{PCl})$}

El PCI es la variable más importante del proceso, pues depende directamente de la composición y mide el contenido energético por unidad de masa o unidad volumen del combustible. Para mezclas gaseosas se define como:

$$
P C I=\sum y_{i} P C I_{i}
$$

Donde $y_{\mathrm{i}}$ es la fracción molar del componente i y $\mathrm{PCI}_{\mathrm{i}}$ es el poder calorífico inferior del componente $\mathrm{i}$.

\section{4 Índice de Wobbe}

Para analizar el efecto del poder calorífico de la mezcla se calculó el índice de Wobbe (IW), el cual es utilizado como un buen indicador de la intercambiabilidad de los combustibles, suministrando una medida indirecta del contenido energético. Se define como:

$$
I W=\frac{P C I}{\sqrt{d_{r}}}
$$

Donde $\mathrm{d}_{\mathrm{r}}$ es la densidad relativa del gas y PCI es el poder calorífico inferior del combustible. 


\subsection{Temperatura adiabática de llama}

La temperatura adiabática de llama es la máxima temperatura que pueden alcanzar los productos de combustión, es decir, aquella que se obtiene si todo el calor generado durante el proceso se utilizará para calentar los productos o asumiendo un proceso adiabático. Esta temperatura fue calculada mediante el software Aspen Hysys 2006.5, asumiendo un proceso adiabático.

\subsection{Eficiencia del proceso}

La eficiencia de combustión en hornos es la medida del calor liberado en la llama que es absorbido por el fluido a calentar; se calcula a partir de las pérdidas de calor en la chimenea y la energía que libera el combustible utilizado. Para su evaluación se utilizó el modelo de entradas y salidas (indirecto) descrito en la norma ASME PTC 4.1 [16]. Se define como:

$$
\eta=\frac{\mathrm{PCI}-Q_{\text {chimenea }} * 100}{\mathrm{PCI}}
$$

Donde PCI es el poder calorífico inferior del combustible y el $Q_{\text {chimenea }}$ es el calor perdido en chimenea.

\subsection{Simulación del proceso}

El proceso de combustión descrito en este trabajo utiliza como cámara de combustión un reactor de conversión tipo "batch". La figura 1 muestra el esquema utilizado para la simulación. En la primera etapa las corrientes de gas combustible (GR o GN) y aire son llevadas a un mezclador con el fin de asegurar una correcta mezcla aire-combustible; entonces la mezcla es enviada al reactor de conversión que se encuentra a presión atmosférica. Inicialmente los productos de combustión salen a condiciones adiabáticas, con el fin de obtener la temperatura adiabática de llama; posteriormente, es retirado el calor necesario para mantener la temperatura de salida del proceso a $320^{\circ} \mathrm{C}$ y de esa manera obtener los productos de combustión a la temperatura de chimenea. Las condiciones de proceso de las corrientes de entrada son mostradas en la tabla 1 .

Tabla 1. Condiciones de los flujos de entrada al proceso

\begin{tabular}{|l|l|l|}
\hline \multicolumn{1}{|c|}{ Corriente } & \multicolumn{1}{|c|}{ Temperatura $\left[{ }^{\circ} \mathrm{C}\right]$} & Presión $[\mathrm{KPa}]$ \\
\hline Combustible & 32,22 & 101,4 \\
\hline Aire & 32,22 & 101,4 \\
\hline
\end{tabular}

Fuente: elaboración propia

Se utilizó un flujo de gas combustible de $1 \mathrm{kmol} / \mathrm{h}$ y el flujo de aire fue variado de acuerdo con el exceso de

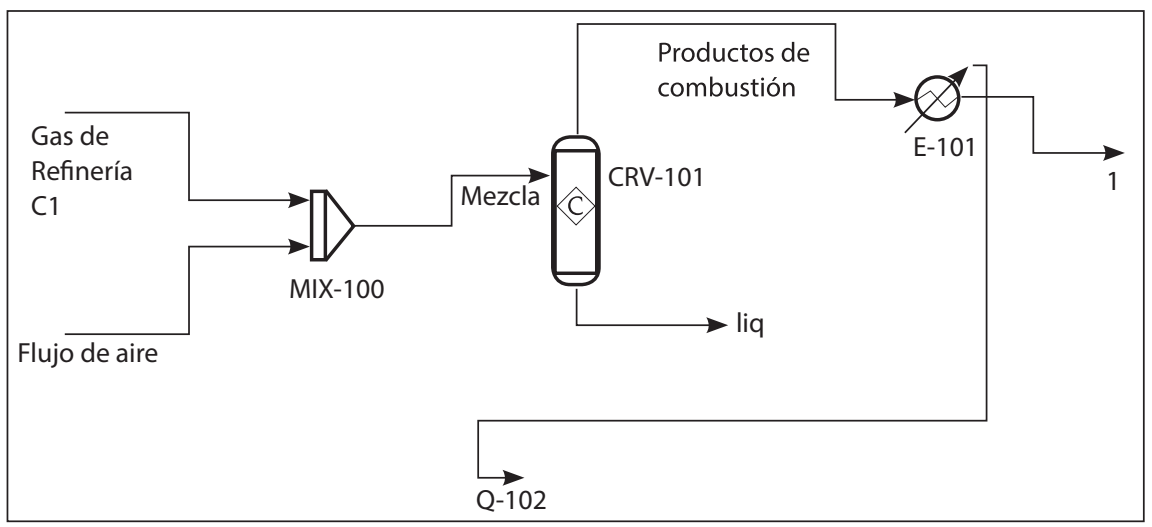

Fig. 1. Simulación del proceso de combustión en Aspen Hysys.

Fuente: elaboración propia. 
oxigeno $\left(\% \mathrm{O}_{2}\right)$ desde $\mathrm{n}=0$, mínimo o estequiométrico, hasta $\mathrm{n}=10 \%$, máximo valor reportado en la literatura.

\subsection{Reacciones de combustión}

A continuación se muestran las reacciones linealmente independientes que se dan en el reactor de conversión.

$$
\begin{aligned}
& \mathrm{CH}_{4}+2 \mathrm{O}_{2} \rightarrow \mathrm{CO}_{2}+2 \mathrm{H}_{2} \mathrm{O}+882,81 \mathrm{Btu} / \mathrm{pie}^{3} \\
& 2 \mathrm{C}_{2} \mathrm{H}_{6}+7 \mathrm{O}_{2} \rightarrow 4 \mathrm{CO}_{2}+6 \mathrm{H}_{2} \mathrm{O}+ \\
& 1580,96 \mathrm{Btu} / \mathrm{pie}^{3} \\
& \mathrm{C}_{3} \mathrm{H}_{8}+5 \mathrm{O}_{2} \rightarrow 3 \mathrm{CO}_{2}+4 \mathrm{H}_{2} \mathrm{O}+ \\
& 2282,34 \mathrm{Btu} / \mathrm{pie}^{3} \\
& n-\mathrm{C}_{4} \mathrm{H}_{10}+\frac{13}{2} \mathrm{O}_{2} \rightarrow 4 \mathrm{CO}_{2}+5 \mathrm{H}_{2} \mathrm{O}+ \\
& 3003,21 \mathrm{Btu} / \mathrm{pie}^{3} \\
& \mathrm{C}_{2} \mathrm{H}_{4}+3 \mathrm{O}_{2} \rightarrow 2 \mathrm{CO}_{2}+2 \mathrm{H}_{2} \mathrm{O}+ \\
& 1462,02 \mathrm{Btu}^{\mathrm{p}} \mathrm{pie}^{3} \\
& \mathrm{C}_{3} \mathrm{H}_{6}+\frac{9}{2} \mathrm{O}_{2} \rightarrow 3 \mathrm{CO}_{2}+3 \mathrm{H}_{2} \mathrm{O}+ \\
& 2146,89 \mathrm{Btu}^{\mathrm{p}} \mathrm{pie}^{3} \\
& 2 \mathrm{H}_{2}+\mathrm{O}_{2} \rightarrow 2 \mathrm{H}_{2} \mathrm{O}+265,40 \mathrm{Btu} / \mathrm{pie}^{3}
\end{aligned}
$$

\section{ANÁLISIS DE RESULTADOS}

En esta sección se analizan los efectos de la composición del gas combustible sobre las características de la combustión, y se evaluó: poder calorífico, índice de wobbe, aire teórico, temperatura adiabática de llama, eficiencia y concentración de $\mathrm{CO}_{2}$ y vapor de agua.

\subsection{Influencia de la composición del gas com- bustible (GR) sobre el poder calorífico de la mezcla}

Para la simulación se tomaron 4 corrientes de
GR obtenidas previamente, mediante un análisis estadístico utilizando Statgraphics, que permitió establecer 8 componentes principales que forman un mezcla representativa de la red de gas combustible con PCI entre $800 \mathrm{Btu} / \mathrm{ft}^{3}$ y $2000 \mathrm{Btu} / \mathrm{ft}^{3}(\mathrm{Cl}$, $\mathrm{C} 2, \mathrm{C} 3$ y C4) y una corriente de gas natural típico Colombiano (tabla 2).

Tabla 2. Composiciones representativas del GR.

\begin{tabular}{|l|c|c|c|c|c|}
\hline \multicolumn{1}{|c|}{ Tipo gas } & GN & C1 & C2 & C3 & C4 \\
\hline CH4 & 97 & 55 & 70 & 25 & 35 \\
\hline C2H6 & 1 & 10 & 0 & 8 & 3 \\
\hline C3H8 & 1 & 0 & 16 & 25 & 35 \\
\hline C4H10 & 0 & 4 & 5 & 10 & 12 \\
\hline C2H4 & 0,5 & 5 & 3 & 10 & 7 \\
\hline C3H6 & 0,5 & 2 & 0 & 5 & 8 \\
\hline H2S & 0 & 4 & 1 & 2 & 0 \\
\hline H2 & 0 & 20 & 5 & 15 & 0 \\
\hline PCI & 913 & 955 & 1200 & 1530 & 1800 \\
\hline
\end{tabular}

Fuente: elaboración propia

La figura 2 muestra la variación del poder calorífico inferior que se presenta al utilizar los 4 tipos de GR a estudiar, calculados mediante la ecuación (1). Se utilizó un rango de PCI entre [800-2000 BTU/pie^3].

El poder calorífico de un combustible depende netamente de su composición, afecta el rendimiento del horno, incluyendo las características de la combustión (longitud de la llama, estabilidad de la llama y temperatura), la eficiencia térmica y las emisiones. Por lo tanto, es un parámetro de vital importancia en el estudio de la combustión [17]. Como se observa en la figura 2 se puede tener un amplio rango en el poder calorífico del GR, donde el GN oscila entre 850-1000 Btu/pie³; asimismo, encontramos GR con PCI entre los 1000-1500 $\mathrm{Btu} / \mathrm{pie}^{3}$, los cuales presentan alto contenido de metano pero se incrementan hidrocarburos como el etano, el etileno y el hidrógeno; también se encuentran GR con PCI superior a los $1500 \mathrm{Btu} / \mathrm{pie}^{3}$, 


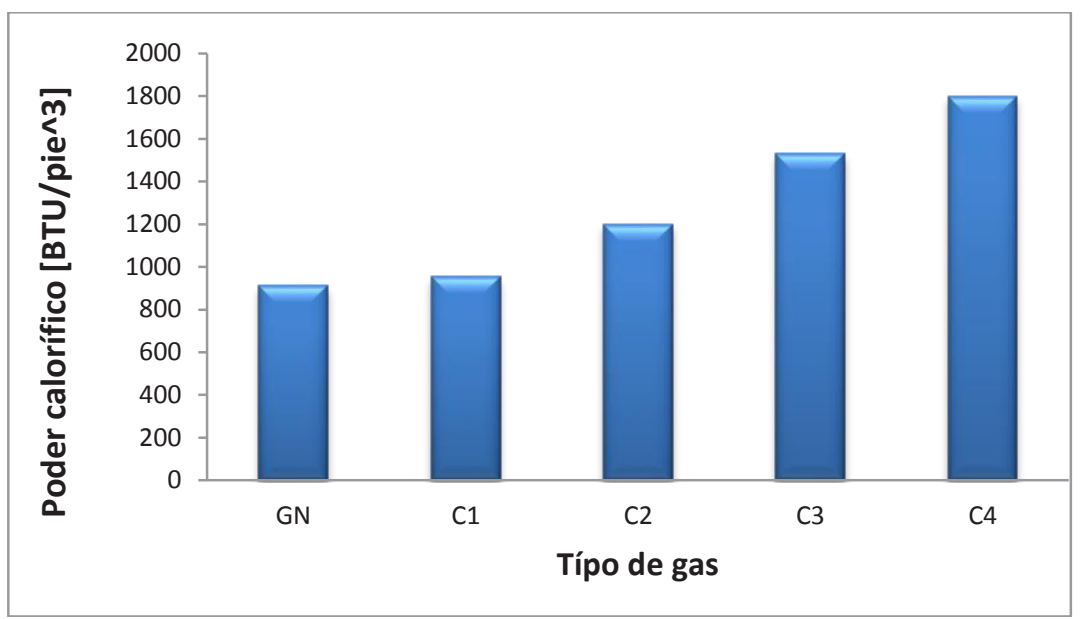

Fig. 2. Poder calorífico inferior de las mezclas combustibles de GR.

Fuente: elaboración propia.

en los cual se presenta bajo contenido de metano, pero se tienen altas concentraciones de propano y butano. De esa forma, se tiene una amplia gama de GR, el cual es utilizado como combustible en las refinerías; de esa manera se hace importante validarlos teniendo en cuenta su intercambiabilidad y los impactos que generan al utilizarse como combustibles.

\section{2 Índice de Wobbe}

El índice de Wobbe se calculó por medio de la ecuación (2). Ya que dicho índice tiene en cuenta el efecto de la composición, y el PCI, se pueden tener dos combustibles con diferentes composiciones y PCI, que puedan tener el mismo valor en el IW;por tanto, presentarían características similares para la combustión y de esa manera se considerarían intercambiables.

En la tabla 3 se observa que el GN y C1 presenta prácticamente el mismo índice de Wobbe, por lo cual se considerarían intercambiables; en el caso de $\mathrm{C} 2, \mathrm{C} 3$ y $\mathrm{C} 4$, sus IW difieren totalmente respecto al del GN por lo no se deben considerar como sustitutos del GN. Sin embargo, dichos gases son utilizados comúnmente en las refinerías; por consiguiente, se analizará el efecto de dicho gases sobre la combustión.

\subsection{Influencia de la composición del GR sobre el exceso de oxígeno}

La cantidad de aire necesario para garantizar la combustión completa para el GN y el GR es calculado mediante la ecuación (11).

$$
A_{0}=\frac{1}{0,21}\left(\begin{array}{l}
2 \mathrm{CH}_{4}+3,5 \mathrm{C}_{2} \mathrm{H}_{6}+5 \mathrm{C}_{3} \mathrm{H}_{8}+ \\
6,5 \mathrm{C}_{4} \mathrm{H}_{10}+3 \mathrm{C}_{2} \mathrm{H}_{4}+4,5 \mathrm{C}_{3} H_{6} \\
+0,5 \mathrm{H}_{2}
\end{array}\right)
$$

Donde $\mathrm{CH}_{4}, \mathrm{C}_{2} \mathrm{H}_{6}, \mathrm{C}_{3} \mathrm{H}_{8}, \mathrm{C}_{4} \mathrm{H}_{10}, \mathrm{C}_{2} \mathrm{H}_{4}, \mathrm{C}_{3} \mathrm{H}_{6}$ y $\mathrm{H}_{2}$ se expresan como la fracción molar del combustible.

En la figura 3 se observa que para $1 \mathrm{kmol} \mathrm{de}$ GN es necesario suministrar 9,82 kmol de aire; al realizar el cambio de combustible se observa que $\mathrm{C1}$ requiere una cantidad menor al GN (1\%), debido a la adición de hidrógeno (20\%), tal como reporta Lee $\mathrm{CH}$ et al. [12], ya que dicho componente disminuye la cantidad de aire, pues requiere $0,5 \mathrm{~mol}$ de oxígeno por mol de combustible, mientras que para C2, C3 y C4 se presenta un aumento del 28, 59 y $89 \%$ en la cantidad de aire, respecto al GN; dicho 
aumento se presenta porque estos gases poseen la mayores concentraciones de propano, butano y propileno, que de acuerdo con las reacciones de combustión $(6,7,9)$, necesitan mayor cantidad de moles de oxigeno por mol de combustible (5 para el propano, 6,5 para el butano y 4,5 para el propileno) para asegurar la combustión completa, comparado con el metano (principal componente del GN) que solo necesita 2 mol de oxígeno. Por tanto, la presencia de hidrocarburos de alto peso molecular en el GR incrementa la cantidad de aire requerido para la combustión, y de ese modo, se corre el riesgo de presentarse combustión incompleta.

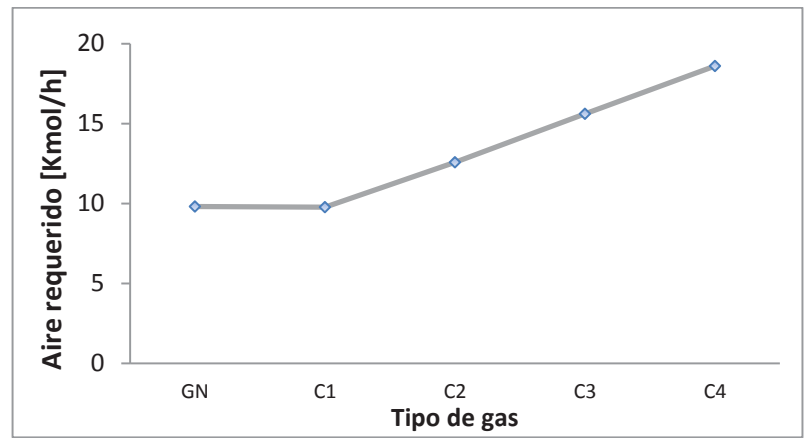

Fig. 3. Aire teórico requerido por el GR.

Fuente: elaboración propia.

\subsection{Influencia de la composición del GR sobre la temperatura adiabática de llama}

La figura 4 muestra la variación de la temperatura adiabática de llama en función del exceso de oxígeno para cada clase de GR y para el GN. Se observa que la máxima temperatura se obtiene para $0 \%$ de exceso de aire, la cual corresponde a la condición estequiométrica para cada uno de los gases, tal como se reporta en la literatura. De igual forma, se observa que a un mismo exceso de aire la temperatura adiabática del GN es de $2036{ }^{\circ} \mathrm{C}$; al usar el GR se obtienen temperaturas adiabáticas superiores que van desde 2066 hasta $2106{ }^{\circ} \mathrm{C}$; estas altas temperaturas hacen que en la zona de reacción se presente combustión a alta temperatura $\left(>1300^{\circ} \mathrm{C}\right)$, la cual favorece los mecanismos de formación del NOx térmico, pues a estas temperaturas, el NOx es iniciado principalmente por la formación de tres radicales libres: nitrógeno, oxígeno y $\mathrm{OH}$, que provienen de la oxidación del nitrógeno circundante bajo el calor liberado durante la combustión, y reaccionan con las moléculas presentes en el aire, llevando a una rápida formación de NOx [9], además, se debe tener en cuenta que las emisiones de NOx aumentan exponencialmente con la temperatura [9]. De igual forma se ha encontrado que las altas temperaturas influyen sobre la vida útil de los hornos, Ul-Hamid et al, mostraron que al exponer el material excesivamente a altas temperaturas (superiores a $900^{\circ} \mathrm{C}$ ), se presentan grietas en la superficie de los tubos como resultado de la aceleración de la carburación por la temperatura [18]; asimismo, se ha encontrado que en los procesos petroquímicos la causa principal de las fallas en los tubos se presenta por corrosión a alta temperatura, pues se genera oxidación, sulfidación, carburación y formación de polvo metálico [19].

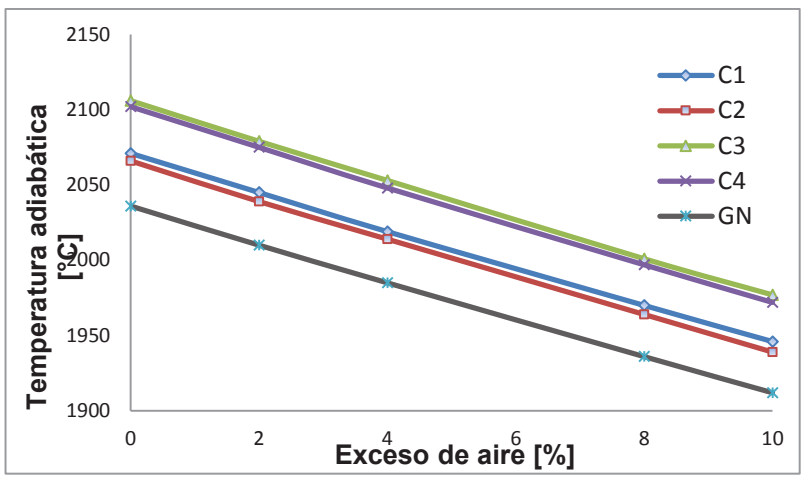

Fig. 4. Efecto del exceso de oxígeno sobre la temperatura adiabática de llama.

Fuente: elaboración propia.

Los resultados también muestran que la temperatura no aumenta directamente con el poder calorífico del gas, pues la máxima temperatura adiabática se obtiene al utilizar C3 [1530 BTU/ pie$\left.^{\wedge} 3\right]$.

En la figura 4 también se observa que al aumentar el exceso de aire, la temperatura adiabática de llama disminuye linealmente a una razón de $12,37^{\circ} \mathrm{C}$ por unidad de aire; por lo tanto, el exceso 
de aire a utilizar no debe ser alto, pues disminuye drásticamente la temperatura causando una disminución en la eficiencia del proceso [15]. Este resultado es similar al reportado por Nabi [20], quien afirma que para motores de combustión, al incrementar el contenido de oxígeno en el combustible se disminuye linealmente la temperatura adiabática de llama, logrando así una disminución en las emisiones de $\mathrm{NO}_{\mathrm{x}}$. De ese modo el exceso de oxígeno debe ser mínimo pero se debe asegurar combustión completa.

\subsection{Influencia de la composición del GR sobre los productos de combustión}

En la figura 5 se muestra la cantidad de $\mathrm{CO}_{2}$ y vapor de agua generado por la combustión completa de los gases estudiados (GN y GR) para un exceso de oxígeno del $2 \%$. Se observa que la concentración molar de $\mathrm{CO}_{2}$ aumenta al utilizar el gas de refinería, desde $9.4 \%$ hasta $11,3 \%$ respecto al GN, lo cual significa un aumento en la generación de emisiones de gases de efecto invernadero. Este aumento en la concentración de $\mathrm{CO}_{2}$ se presenta debido al alto contenido de propano y butano en el GR, pues dichos hidrocarburos emiten más $\mathrm{CO}_{2}$ que el metano, tal como muestran las reacciones de combustión (4-10); por tanto, a mayor contenido de propano y butano en el GR se generarán mayores emisiones de $\mathrm{CO}_{2}$, convirtiéndose en una desventaja ambiental.

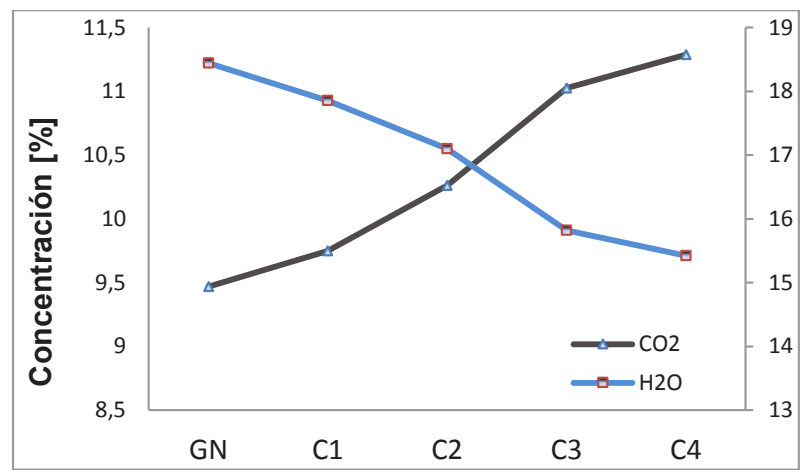

Fig. 5. Efecto del GR sobre la composición de los productos de combustión.

Fuente: elaboración propia.

\subsection{Influencia de la composición GR sobre la eficiencia del proceso}

En la figura 6 se muestra la variación de la eficiencia del proceso de combustión en función del tipo de gas (GR y GN) utilizado, para un exceso de oxígeno del $2 \%$. Se observa que la eficiencia disminuye respecto al GN para los gases $\mathrm{C} 1, \mathrm{C} 2$ y C3, donde $\mathrm{C} 1$ presenta una disminución significativa del $2 \%$, atribuido a la presencia de hidrógeno en el combustible [12]; en el caso de C2 y C3 la disminución es muy baja, por lo cual dichos gases no generarían impactos negativos sobre la eficiencia; finalmente en el caso de C4 se logra un aumento del 0,4\%, debido a la presencia de hidrocarburos de alto poder calorífico y a la ausencia de hidrógeno.

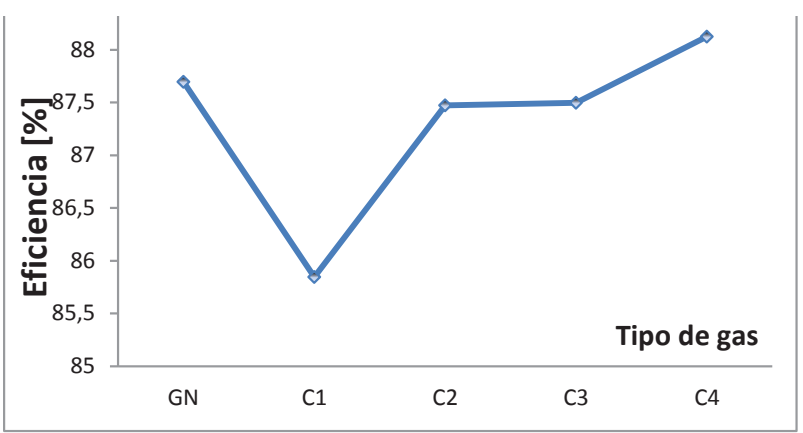

Fig. 6. Efecto del GR sobre la eficiencia del proceso Fuente: elaboración propia.

\section{CONCLUSIONES}

La simulación de las mezclas combustibles de GR en Aspen Hysys ayudó a valorar las características de la combustión, mediante la variación de la composición del combustible, permitiendo establecer las variables que afectan la combustión. De esa manera se encontró que la presencia de hidrocarburos de alto peso molecular en el GR incrementa la cantidad de aire a utilizar para asegurar la combustión completa, y aumenta las emisiones de $\mathrm{CO}_{2}$ convirtiéndose en una desventaja ambiental.

Igualmente el GR aumenta la temperatura adiabática de llama, causando de esa forma un aumento en las emisiones de NOx y de igual forma 
favorece los mecanismos de falla por corrosión a alta temperatura; sin embargo, no se encontró una relación directa entre la temperatura y el PCI del GR. Por otra parte las mezclas combustibles evaluadas presentan una variabilidad en la eficiencia respecto al GN, disminuyendo con el aumento en la concentración de hidrógeno.

Finalmente los resultados de este estudio ponen en evidencia la variabilidad en la temperatura adiabática, eficiencia y emisiones que se presenta al utilizar el GR como combustible, sin embargo es posible encontrar rangos de composición en los cuales se pueda hacer un mejor uso del GR, que maximice el uso de energía y minimice la generación de contaminantes.

\section{AGRADECIMIENTOS}

Agradecemos a Colciencias por el apoyo económico brindado a este proyecto a través del Programa Jóvenes investigadores e innovadores, año 2011, a la Universidad Industrial de Santander y a Ecopetrol - Instituto Colombiano de Petróleo por el apoyo general que ha hecho posible el desarrollo de este estudio.

\section{REFERENCIAS}

[1]. J.L. Hu, C. H. Kao, "Efficient energy-saving targets for APEC economies”. Energ. Pol, vol. 35, pp. 373-82, 2007.

[2]. P. Lunghi, R. Burzacca, "Energy recovery from industrial waste of a confectionery plant by means of BIGFC plant”. Energy, vol. 29, pp. 2601-17, 2004.

[3]. A. AMELL, "Tecnología de la Combustión de Gases y Quemadores Atmosféricos de Premezcla”. [En línea], Disponible: http://es.scribd.com/ doc/73707395/3-Quemadores-Atmosfericos-1,1996.

[4]. Wobbe, G., "A new definition of the Quality of gas". L'Industria de Gas e degli Acquedotti, vol. 30, 1926.

[5]. American Gas Association Laboratories, "Interchangeability of Other Fuel Gases with Natural Gases, Research Bulletin Number 36". AGA Committee on Mixed Gas Research, Joint Committee of Natural Gas Department and Technical Section, American Gas Association, 1946.

[6]. P. Delbourg, H. Schneck, "Interchangeability of Gases". Presentado en $47^{\text {th }}$ Congress of I'Industrie du Gaz, Paris, 1957.

[7]. E. R. Weaver, "Formulas and Graphs for Representing the Interchangeability of Fuel Gases". Journal of Research of the National Bureau of Standards, vol. 46, n. $3,1951$.

[8]. S.C. Hsieh, C. J. G. Jou, "Reduction of greenhouse gas emission on a medium-pressure boiler through hydrogen-rich fuel control”. Appl. Therm. Eng, vol. 27, pp. 2924-2928, 2007.

[9]. C. L. Lee, C. J. G. Jou, H. S. Tai, C.H. Wang, S.C. Hsieh, H. P. Wang, "Reduction of nitrogen oxides emissions of a medium-pressure boiler through fuel control”. Aerosol Air Qual. Res, vol. 6, n. 2, pp. 123-133, 2006.

[10]. C. J. G. Jou, C. L. Lee, C. H. Tsai, H. P. Wang, "Enhancing the performance of a high-pressure cogeneration boiler with waste hydrogen-rich fuel”. Inter. Jour. Hydrogen Energy, vol. 33, pp. 5806-5810, 2008.

[11]. A. M. Mastral, R. Murillo, T. García, M. V. Navarro, M. S. Callen, J. M. López, "Study of the viability of the process for hydrogen recovery from old tyre oils”. Fuel Processing Technology, vol. 75, pp. 185-199, 2002.

[12]. C. L. Lee, S. S. Hou, W. J. Lee, C. J. Jou, "Improving cost-effectiveness for the furnace in a full-scale refinery plant with reuse of waste tail gas fuel”. International 
Journal of Hidrogen Energy, vol. 35, pp. 1797-1802, 2010.

[13]. T. Rogaume, M. Auzanneau, F. Jabouille, C. J. Goudeau, J. L. Torero, "The effects of different airflows on the formation of pollutants during waste incineration". Fuel, vol. 81, pp. 2277-2288, 2002.

[14]. F. Wildy, "Fired heater optimization" Technical sales support manager, AMETEK process instruments, Pittsburgh, PA 15044.

[15]. J. Serrano, Y. Carranza, "Análisis teórico de la combustión en quemadores de gas natural". Scientia et Technica, vol. 29, pp. 139-143, 2005.

[16]. ASME, Fired Steam Generators, Performance Test Code 4, ASME PTC 4.1, 1998.

[17]. Y. C. Ko, T. H. Lin, "Emissions and efficiency of a domestic gas stove burning natural gases with various compositions". Energy Conversion and Management, vol. 44, pp. 3001-3014, 2003.

[18]. A. Ul-Hamid, H. M. Tawancy, A. R. Mohammed, M. A. Nureddin, "Failure analysis of furnace radiant tubes exposed to excessive temperature". Engineering Failure Analysis, vol. 13, pp. 1005-1021, 2006.

[19] J. Serna, "Oxidación, carburación y sulfidación de aleaciones ferríticas Fe-9Cr-1Mo modificadas en ambientes con hidrocarburos a temperaturas entre 550 y $750^{\circ} \mathrm{C}$. Tesis de Doctorado en Ingeniería Química, Universidad Industrial de Santander. Bucaramanga. 2004.

[20]. M. N. Nabi, "Theoretical investigation of engine thermal efficiency, adiabatic flame temperature, NOx emission and combustion-related parameters for different oxygenated fuels". Applied Thermal Engineering, vol. 30, pp. 839-844, 2010. 